\title{
Kosten und Benützung städtischer Infrastrukturen am Beispiel von Bern
}

\author{
(Die Verflechtungen einer Kernstadt mit den Außengemeinden der Region)
}

\section{Einleitung}

\subsection{Zweck der Untersuchung, Vorgehen}

Die Kernstädte der schweizerischen Agglomerationen erbringen seit jeher, vor allem aber in den letzten Jahrzehnten - bedingt durch die starken Entmischungsprozesse der Funktionen Wohnen, Arbeiten und Erholung - große Infrastrukturleistungen, die aber weitgehend von einer in den letzten Jahren abnehmenden Zahl von Bewohnern der Kernstädte getragen werden müssen. Der Neuverteilungsprozeß der Lasten ist je nach Infrastrukturgebiet und Stadt schon unterschiedlich weit fortgeschritten oder hat noch gar nicht begonnen.

Das Statistische Amt der Stadt Bern wurde 1978 mit der Durchführung einer Untersuchung über die Verflechtungen der Kernstadt mit den Außengemeinden der Region Bern beauftragt. Neben den Mitarbeitern des Amtes unter Leitung von Dr. rer. pol. ANDREAS KNECHT wurden für spezielle Fragen externe Fachleute beigezogen. Die Studie ist somit eine interdisziplinäre Teamarbeit zwischen Statistiker, Volksund Betriebswirtschafter und Geograph.

Ausgangspunkt der Untersuchungen bildete die Gemeinderechnung der Stadt Bern für das Jahr 1977. Dazu kamen eine große Zahl von Einzelauskünften, Ergänzungen und Erhebungen bei verschiedenen Amtsstellen und Auskunftspersonen (siehe detailliertes Literatur- und Quellenverzeichnis der Studie).

Die Untersuchung der Verflechtungen der Stadt Bern soll sich aber nicht nur auf den Bereich der Infrastruktur beschränken, sondern soll auch auf die Einkommens- und Konsumströme und deren externe Effekte auf die Finanzen der öffentlichen Hand erweitert werden. Schließlich soll der Versuch einer Leistungsbilanz mit Gegenüberstellung aller positiven und negativen externen Effekte gemacht werden. Der Infrastrukturteil der Studie ist abgeschlos-

1) MESSMER, O., Orts- und Regionalplanung, Grundlagen, Grundbegriffe und wirtschaftliche Aspekte. Skriptum Uni Bern, Wintersemester 1971/72, S. 22, Bern 1971.

2) vergleiche: Studienkommission für Preis-, Kosten- und Strukturfragen, Studien zur Regionalpolitik. Bericht zu Handen des Eidg. Volkswirtschaftsdepartementes, Bern 1972, S. 28 ff. sen, während die weiteren Untersuchungen im jetzigen Zeitpunkt noch laufen.

\subsection{Begriffsdefinitionen}

In diesem Abschnitt sollen zwei zentrale Begriffe der Studie definiert werden: Infrastruktur und externe Effekte.

Wir verstehen unter dem Begriff Infrastruktur diejenigen Güter und Dienstleistungen, die zur Ermöglichung und Förderung aller Lebensäußerungen des Menschen notwendig sind und nicht von ihm selbst, sondern durch die Öffentlichkeit auf Grund eines politischen Entscheids bereitgestellt werden. ${ }^{1)}$

Als externe Effekte bezeichnen wir allgemein Nutzen und Kosten, bei denen Verursacher und Träger nicht identisch sind und kein Markt- bzw. Tauschverhältnis besteht. ${ }^{2)}$ Bezogen auf die einzelnen Gemeinden einer Region können wir von räumlichen externen Effekten der Nutzen bzw. Kosten öffentlicher Leistungen sprechen. Diese räumlichen externen Effekte werden auch als Spillovers bezeichnet. Wir betrachten also im folgenden die räumlichen externen Effekte der öffentlichen Leistungen einer Gemeinde auf Einwohner und Unternehmungen anderer Gemeinden, wobei wir die Zuordnung gemäß Steuerdomizil der betreffenden natürlichen oder juristischen Personen vornehmen.

\subsection{Die Region als Wirtschaftseinheit}

Unter dem Begriff Region Bern ist im folgenden das Gebiet der Partnergemeinden des Regionalplanungsvereins Stadt Bern und umliegende Gemeinden zu verstehen, welches wir in Kernstadt (Einwohnergemeinde Bern) und Außengemeinden (25 Einwohner- und Viertelsgemeinden, siehe Fig. 1) unterteilen. Die Region Bern bildet heute eine Wirtschaftseinheit, welche durch zahlreiche Verflechtungen zwischen Kernstadt und Außengemeinden gekennzeichnet ist.

Die Kernstadt hätte ihre heutige Bedeutung ohne das Einzugsgebiet in den Außengemeinden nicht erlan-

\section{Dr. Ernst Gächter, Statistisches Amt der Stadt Bern,} Gutenbergstrasse 27, 3011 Bern. 


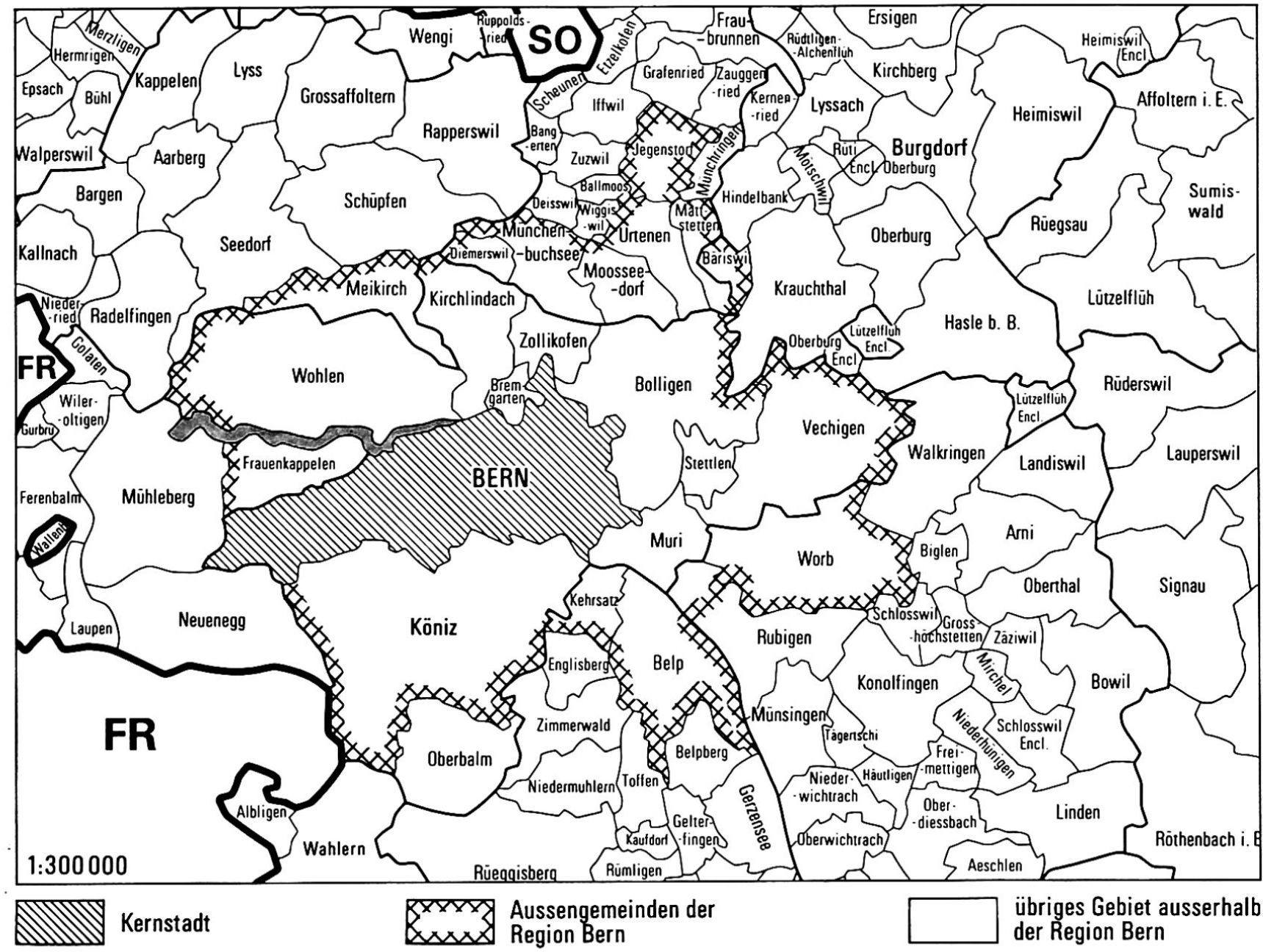

gen können; diese andererseits verdanken ihr Wachstum den Impulsen aus der Kernstadt. In Tabelle 1 sind einige Daten zur Entwicklung von Wohnbevölkerung, Wohnungen und Arbeitsplätzen zusammengestellt. Während die Stadt Bern seit 1962 ständig rückläufige Bevölkerungszahlen ausweist, zeigen die Außengemeinden bis 1973 ein rasches, seither ein gebremstes Wachstum. 1960 hatten noch beinahe zwei Drittel aller Regionsbewohner ihren Wohnsitz in der Stadt Bern, Ende 1979 wohnten bereits über $51 \%$ in den Außengemeinden. Von ebenso großer Bedeutung wie die Entwicklung der absolu-

Tabelle 1 Region Bern: Entwicklung einiger sozio-ökonomischer Merkmale seit 1960

\begin{tabular}{|c|c|c|c|c|c|c|}
\hline Merkmal & Jahr & $\begin{array}{l}\text { Stadt Bern } \\
\text { absolut }\end{array}$ & in $\%$ d. Region & $\begin{array}{l}\text { Außenge } \\
\text { absolut }\end{array}$ & $\begin{array}{l}\text { meinden }{ }^{1)} \\
\text { in } \% \mathrm{~d} \text {. Region }\end{array}$ & Region Bern ${ }^{1)}$ \\
\hline Wohnbevölkerung & $\begin{array}{l}1960 \\
1970 \\
1975 \\
1979\end{array}$ & $\begin{array}{l}163172 \\
162405 \\
149834 \\
141303\end{array}$ & $\begin{array}{l}64,7 \\
55,9 \\
51,0 \\
48,4\end{array}$ & $\begin{array}{r}88837 \\
128084 \\
143830 \\
150513\end{array}$ & $\begin{array}{l}35,3 \\
44,1 \\
49,0 \\
51,6\end{array}$ & $\begin{array}{l}252009 \\
290489 \\
293664 \\
291816\end{array}$ \\
\hline Wohnungsbestand & $\begin{array}{l}1960 \\
1970 \\
1979\end{array}$ & $\begin{array}{l}53544 \\
61986 \\
69434\end{array}$ & $\begin{array}{l}68,8 \\
60,1 \\
55,1\end{array}$ & $\begin{array}{l}24335 \\
41187 \\
56614\end{array}$ & $\begin{array}{l}31,2 \\
39,9 \\
44,9\end{array}$ & $\begin{array}{r}77879 \\
103173 \\
126048\end{array}$ \\
\hline Beschäftigte & $\begin{array}{l}1960 \\
1970 \\
1975\end{array}$ & $\begin{array}{r}99097 \\
118051 \\
122418\end{array}$ & $\begin{array}{l}78,8 \\
76,7 \\
76,9\end{array}$ & $\begin{array}{l}26705 \\
35957 \\
36703\end{array}$ & $\begin{array}{l}21,2 \\
23,3 \\
23,1\end{array}$ & $\begin{array}{l}125802 \\
154008 \\
159121\end{array}$ \\
\hline
\end{tabular}

1) alle Zahlen ohne Viertelsgemeinde Allmendingen 
ten Bevölkerungszahlen sind die Änderungen im Aufbau der Bevölkerung von Stadt Bern und AuBengemeinden (Altersstruktur, Einkommensstruktur z. B.).

Die Zahl der Arbeitsplätze stieg in der Stadt Bern von rund $100000 \mathrm{im}$ Jahre 1960 und $118000 \mathrm{im}$ Jahre 1970 auf ein geschätztes Maximum von 127000 im Jahre 1973. Seither fiel die Zahl über rund $122000 \mathrm{im}$ Jahre 1975 auf den geschätzten Wert von 115000 im Jahre 1977. Die hohe Relation Arbeitsplätze/Wohnbevölkerung in der Stadt Bern (1977 79 Arbeitsplätze auf 100 Personen der Wohnbevölkerung) wurde durch die tägliche Pendelwanderung zwischen auswärtigem Wohnort und Arbeitsort Bern ermöglicht. Die Zahl der Pendler erhöhte sich dabei nicht allein wegen der steigenden Zahl der Arbeitsplätze in der Stadt Bern, sondern auch wegen der abnehmenden Zahl in der Kernstadt wohnhafter Erwerbstätiger. Zwischen 1960 und 1977 hat sich die Anzahl der Zupendler aus den Außengemeinden von 14077 auf rund 31500 mehr als verdoppelt. 1977 wurde bei rund 5000 Wegpendlern aus der Stadt Bern die Zahl der Zupendler in die Stadt Bern insgesamt auf 47000 geschätzt (15 500 Zupendler von außerhalb der Region Bern).

\subsection{Die finanzielle Lage der Regionsgemeinden}

Während 1960 (bei einer Steueranlage von 2,0 in der Stadt Bern) 21 Außengemeinden eine höhere und nur drei die gleiche oder eine tiefere Steueranlage hatten, wiesen 1979 (Steueranlage der Stadt Bern 2,3) nur noch acht Außengemeinden eine höhere, sechs die gleiche und bereits zehn eine tiefere Steueranlage auf.

Auch die absolute Steuerkraft (Ertrag aus den Einkommens- und Vermögenssteuern der natürlichen Personen, den Gewinn- und Kapitalsteuern der juristischen Personen und den Liegenschaftssteuern bei der Anlage von 1,0) von Stadt Bern und Außengemeinden hat sich stark verändert: Die Stadt Bern konnte ihre absolute Steuerkraft von 1960 bis 1977 auf das 4,5fache steigern, die Außengemeinden zusammen aber sogar auf das neunfache. Die relative Steuerkraft (absolute Steuerkraft pro Kopf der Wohnbevölkerung) der Stadt Bern betrug 1960 170,3 Franken, 1977 863,7 Franken, diejenige der Außengemeinden im Durchschnitt 1960 117,5 Franken und 1977 637,9 Franken. Die Steigerung der relativen Steuerkraft der Außengemeinden von 1960 bis 1977 und das Niveau 1977 sind in Anbetracht der niedrigeren Erwerbsquote und der geringeren Zahl von Arbeitsplätzen gegenüber der Kernstadt beträchtlich.

Einem Vergleich der finanziellen Lage der Regionsgemeinden aufgrund der Steuerkraft müssen aber Angaben über die unterschiedliche Belastung durch die Bereitstellung der Infrastruktur gegenüberge- stellt werden. Infolge der Funktionsteilung zwischen den Gemeinden ist die Kernstadt zur Vorhaltung infrastruktureller Leistungen zugunsten der Außengemeinden gezwungen (Details siehe Abschnitt 2). Ein Vergleich der Ausgaben pro Kopf der Wohnbevölkerung zwischen der Kernstadt und den drei größten Außengemeinden (Köniz, Bolligen und Muri mit $52 \%$ der Gesamtbevölkerung der Außengemeinden) im Jahre 1976 ergibt, daß die Kernstadt pro Kopf rund doppelt so hohe Ausgaben ausweist (3785 Franken) als die drei betrachteten Gemeinden (1798 bis 1983 Franken). Einzig im Aufgabengebiet Unterricht und Forschung weist eine Gemeinde einen leicht höheren Wert auf als die Kernstadt. In den übrigen Bereichen hat die Kernstadt durchwegs beträchtlich höhere Pro-Kopf-Ausgaben als die drei Vergleichsgemeinden, z. B. Rechtspflege/Polizei/ Feuerwehr: 296 Franken bzw. 32 bis 71 Franken; Kultur: 131 Franken bzw. 4 bis 22 Franken; Verkehr/Energie: 329 Franken bzw. 93 bis 143 Franken. Die Zahlen vermitteln einen Hinweis auf die Lasten der Kernstadt. Infolge des Bevölkerungsrückganges verteilen sich diese Lasten auf eine immer kleiner werdende Zahl von Einwohnern, was einen überproportionalen Anstieg der Pro-Kopf-Ausgaben bewirkt (Vergleich 1970-1976). Die Steuerkraft pro Kopf dagegen zeigte in der Kernstadt seit 1970 ein gegenüber dem regionalen Mittel unterdurchschnittliches Wachstum. Sowohl bei den Einnahmen wie bei den Ausgaben ergibt sich somit eine negative Entwicklung für die Kernstadt.

\section{Externe Effekte durch Bereitstellung der Infrastruktur}

\subsection{Allgemeine Bemerkungen}

Den im folgenden angeführten Zahlen liegen im allgemeinen die Werte des Jahres 1977 zu Grunde. Die Analyse der einzelnen Infrastrukturleistungen wird nach folgendem Schema durchgeführt:

- Darstellung der realen Verflechtungen,

- Darstellung der finanziellen Verflechtungen,

- Darstellung der externen Effekte.

Die Betrachtung der realen Veflechtung dient der Bestimmung der Schlüsselgröße, nach der die Kosten der Infrastrukturleistungen auf die Nutznießer verteilt werden können. Soweit möglich werden dazu die effektiven Benützerzahlen oder repräsentative Hilfsgrößen verwendet, deren Aufteilung in Kernstadt und Außengemeinden bekannt ist.

Bei der Darstellung der finanziellen Verflechtung wird zuerst die Verteilungsgröße, d.h. der zu verteilende Betrag bestimmt. Als Berechnungsgrundlage werden dabei je nach Sachgebiet die Gesamtkosten (Bruttokosten abzüglich Beiträge Dritter) oder das von der öffentlichen Hand zu tragende Defizit be- 
nutzt. In diesen Beträgen sind Abschreibungen und Verzinsung des investierten Kapitals enthalten. Die Gesamtkosten bzw. das Defizit (Verteilungsgröße) werden dann gemäß dem Durchschnittskostenprin$z i p^{1)}$ der Schlüsselgröße entsprechend anteilsmäßig auf die nutznießenden Gemeinden aufgeteilt.

Durch die Gegenüberstellung dieser rechnerisch ermittelten Sollzahlen mit den Ist-Werten, d.h. den aufgrund bestehender Beitragsregelungen effektiv geleisteten Beiträgen, lassen sich schließlich die externen Effekte speziell zwischen der Kernstadt und den Außengemeinden der Region Bern, zum Teil aber auch zwischen der Kernstadt und ihrem weiteren Einzugsgebiet bestimmen.

Die folgenden Darstellungen beschränken sich auf Infrastrukturbereiche, deren Nutzen der Kernstadt und den Außengemeinden gemeinsam zukommen. ${ }^{2)}$ Dabei sollen sowohl die aus der Bereitstellung dieser Infrastrukturen erwachsenden Belastungen wie die Entlastungen gezeigt werden. Behandelt werden jedoch nur die direkten Kosten, die in den Rechnungen der öffentlichen Hand oder der Privaten erscheinen. Vernachlässigt werden müssen indirekte Kosten wie Lärmbelästigung u.a.

1) Da die Infrastrukturbenützung durch Personen von außerhalb der Stadt Bern ein beträchtliches Ausmaß erreicht (Privatverkehr $58 \%$, Kultur $30-60 \%$, Berufs- und höhere Schulen bis $80 \%$ usw.), wird das Durchschnittskostenprinzip angewandt. Das Grenzkostenprinzip kann unseres Erachtens nur bei kleinen Anteilen von etwa 5 bis $20 \%$ zur Anwendung gelangen.

2) Nicht in Betracht gezogen werden Infrastrukturleistungen der Stadt Bern, die nur von der städtischen Bevölkerung benutzt werden oder deren Kosten durch die Einnahmen voll gedeckt sind (Primar- und Sekundarschulen; Kehrichtbeseitigung, Schlachthof u.a.).

\subsection{Privatverkehr}

Die Berechnungen für den Individualverkehr basieren auf der Straßenrechnung des Bundesamtes für Statistik des Jahres 1976. Diese umfaßt folgende Ausgabenposten: Verwaltung, Personalvorsorge und Sozialversicherung, Unterhalt und Reinigung, Verbesserung und Ausbau, Neubauten, Verkehrssignalisation und -regelung. Den Gesamtkosten der Gemeinde stehen Einnahmen für Straßenzwecke (Gebühren, Beiträge des Bundes, des Kantons und anderer Gemeinden, Beiträge von öffentlichen Korporationen, Bahn- und PTT-Verwaltung sowie Beiträge von Privaten) gegenüber, die auf Gemeindeebene aber nur einen geringen Teil der Kosten decken. Der verbleibende Rest muß aus allgemeinen Mitteln aufgebracht werden (siehe Tab.2). In der Straßenrechnung werden $70 \%$ der Kosten für die Gemeindestraßen als durch den Motorfahrzeugverkehr bedingt angenommen.

Von den rund 280000 Fahrten privater Motorfahrzeuge an einem mittleren Werktag entfallen nach Unterlagen des Stadtplanungsamtes $42 \%$ auf Fahrzeuge aus der Kernstadt, $38 \%$ auf solche von den Außengemeinden und 20\% auf solche von außerhalb der Region Bern. Damit entstehen der Stadt Bern externe Kosten von 15,138 Mio Franken. Der externe Nutzen der Stadt Bern aus Fahrten auf dem Straßennetz der Außengemeinden beläuft sich auf 3,465 Mio Franken. Per Saldo ergibt sich somit für die Stadt Bern eine Belastung von 6,453 Mio Franken im Verhältnis zu den Außengemeinden der Region Bern. Erfaßt sind damit aber nur die direkten Kosten des Privatverkehrs. Dieser dürfte daneben aber auch noch beträchtliche indirekte Auswirkun-

Tabelle 2 Straßenrechnung 1976 und externe Effekte des Privatverkehrs

\begin{tabular}{|c|c|c|c|}
\hline Merkmal & & Stadt Bern & $\begin{array}{l}\text { Außengemeinden } \\
\text { der Region }\end{array}$ \\
\hline Straßenlänge in km (Gemeindestraßen) & & 346,8 & 854,2 \\
\hline Ausgaben für Gemeindestraßen & $\begin{array}{l}\text { - absolut Mio Fr. } \\
\text { - pro Kopf Fr. }\end{array}$ & $\begin{array}{l}42,590 \\
287\end{array}$ & $\begin{array}{l}21,329 \\
147\end{array}$ \\
\hline Einnahmen für Straßenzwecke & $\begin{array}{l}-\quad \text { Mio Fr. } \\
- \text { in } \% \text { der Ausgaben }\end{array}$ & $\begin{array}{l}5,306 \\
12,5\end{array}$ & $\begin{array}{l}4,827 \\
22,6\end{array}$ \\
\hline Aus allgemeinen Mitteln finanziert & $\begin{array}{l}\text { - absolut Mio Fr. } \\
\text { - pro Kopf Fr. }\end{array}$ & $\begin{array}{l}37,284 \\
251\end{array}$ & $\begin{array}{l}16,502 \\
114\end{array}$ \\
\hline Anteil motorfahrzeugbedingte Kosten $(70 \%)$ & Mio Fr. & 26,099 & 11,551 \\
\hline Verkehrsaufkommen nach Herkunft in $\%$ : & $\begin{array}{l}\text { Stadt Bern } \\
\text { Außengemeinden } \\
\text { außerhalb Region Bern }\end{array}$ & $\begin{array}{l}42 \\
38 \\
20\end{array}$ & $\begin{array}{l}30 \\
60 \\
10\end{array}$ \\
\hline Externe Kosten (Mio Fr.) durch Fahrzeuge von: & $\begin{array}{l}\text { Stadt Bern } \\
\text { Außengemeinden } \\
\text { außerhalb Region Bern }\end{array}$ & $\begin{array}{l}9,918 \\
5,220\end{array}$ & $\begin{array}{l}3,465 \\
\dot{1}, 155\end{array}$ \\
\hline
\end{tabular}


gen auf die Stadtentwicklung haben, wohnten doch 1976 rund 15\% der städtischen Einwohner an Straßen, deren Lärmbelastung bei Spitzenverkehr als übermäßig zu qualifizieren war.

\subsection{Kultur}

Einwohner- und Burgergemeinde hatten 1978 Nettoaufwendungen von über 22 Mio Franken zugunsten von kulturellen Institutionen in der Stadt Bern, während die Außengemeinden an einzelne Institutionen aufgrund von Abmachungen oder freiwillig Beiträge von knapp 600000 Franken entrichteten. Außerdem beteiligt sich der Kanton Bern an den finanziellen Lasten der Stadt Bern im Sektor Kulturförderung mit rund 9 Mio Franken.

Die größten Nettoaufwendungen von Einwohnerund Burgergemeinde entfielen auf Stadtheater (7,052 Mio Franken), Berner Orchesterverein/Symphoniekonzerte $(3,590)$, Bernische Musikgesellschaft/Konservatorium $(2,005)$, Stadt- und Universitätsbibliothek $(1,855)$, Naturhistorisches Museum $(1,838)$, Historisches Museum $(1,574)$, Volksbücherei $(1,012)$ und Kunstmuseum $(0,765$ Mio Franken). Im weiteren erhielt eine größere Zahl von kleineren Institutionen noch zusammen 2,442 Mio Franken. Wie hoch sind nun die Benützungsanteile dieser Institutionen durch Auswärtige? Hier standen vielfach keine umfassenden Angaben zur Verfügung, sondern es mußte auf die Anteile bei den Abonnenten u.ä. abgestellt werden. Für die Berechnung der externen Effekte wurden folgende Anteile der Außengemeinden bzw. der Gebiete außerhalb der Region Bern angewendet:

Stadtheater $28,8 \% / 29,8 \%$, Symphoniekonzerte $26,6 \% / 14,8 \%$, Konservatorium $32,2 \% / 16,1 \%$, Stadt- und Universitätsbibliothek $16,2 \% / 32,9 \%$, Museen 25\%/20\% usw. Unter Anwendung dieser Anteile ergab sich ein Betrag von 5,8 Mio Franken für die Außengemeinden und von 4,8 Mio Franken für die Gebiete außerhalb der Region Bern. Diesen Kosten standen Beiträge der betr. Gebiete an diese Leistungen von 596000 bzw. 40000 Franken gegenüber. Damit verbleiben als externer Effekt eine Nettobelastung für die Stadt Bern im Sektor Kultur von insgesamt 10,009 Mio Franken (5,220 Mio Franken Außengemeinden, 4,789 Mio Franken Gebiet außerhalb der Region Bern).

\subsection{Schulen}

Die Stadt Bern führt eine größere Zahl von Schulen (Mittel- und Berufsschulen), deren Einzugsgebiet die gesamte Region umfaßt und zum Teil sogar noch wesentlich darüber hinaus reicht. Als Schlüsselgröße kann bei den Schulen die Herkunft der Schüler verwendet werden. An der Kunstgewerbeschule Bern z. B. ergaben sich im Schuljahr 1977/78 folgende
Anteile nach dem Wohnort der Lehrlinge: Stadt Bern 16,4\%, Außengemeinden 16,6\%, übriger Kanton Bern 42,3\%, andere Kantone 24,7\%. Für die Gymnasien lauteten die Schüleranteile: Stadt Bern $39,9 \%$, Außengemeinden $43,4 \%$ und übriger Kanton Bern 16,6\%.

Wohl leisten Bund und Kanton Bern und in den meisten Fällen aufgrund von kantonalen Vorschriften auch die Wohnsitzgemeinden der Schüler Beiträge an die Schulkosten, doch verbleibt der Stadt Bern als Standortgemeinde je nach Schule eine ansehnliche Nettobelastung. Bei der Bemessung der Kantonsund Wohngemeindebeiträge nimmt der Kanton Bern einen Vorausabzug von den Nettokosten zu Lasten der Standortgemeinde vor. Der Abzug wird mit dem Vorteil der Steuereinnahmen aus den Lehrergehältern und der außerschulischen Benützungsmöglichkeit der Gebäude begründet. Diese Abzüge scheinen uns nicht mehr gerechtfertigt, da heute über $50 \%$ der betr. Lehrer ihren Wohnsitz außerhalb der Stadt Bern haben. Sie werden deshalb in der Studie nicht vorgenommen.

Die Schulkosten werden für jede Schule bzw. jede Ausbildungsart nach dem folgenden Schema berechnet:

Totalaufwand (Besoldungen, Anschaffungen, Verbrauchsmaterial gemäß Gemeinde- bzw. Schulrechnung, Anteil an allgemeine Verwaltungs- und Betriebskosten, Abschreibungen auf Nettoanlagekosten, Verzinsung Restbuchwert der Nettoanlagekosten)

minus Schul- und Kursgeld (sofern sie von allen Schülern in der gleichen Höhe bezahlt werden müssen), Materialgelder, Erträge aus Verkäufen minus Bundesbeiträge (Berufsschulen), Kantonsbeiträge

$=$ Nettoaufwand (Verteilungsgröße).

Die Verteilungsgröße wird prozentual zur Schlüsselgröße auf Stadt Bern, Außengemeinden und Gebiet außerhalb der Region Bern verteilt. Von dem resultierenden Betrag werden noch die Schulkostenbeiträge der Gemeinden und die Schul- und Kursgelder (sofern sie nur von auswärtigen Schülern entrichtet werden müssen) abgezogen. Der verbleibende Betrag ist die Nettobelastung der Stadt Bern zugunsten der Außengemeinden bzw. des Gebietes außerhalb der Region Bern. Da die Schulkostenbeiträge der Wohngemeinden auf einer gesamtkantonalen Basis berechnet werden und zudem nach der Steuerkraft der Wohngemeinden abgestuft sind, fällt der ungedeckte Betrag je nach Kostenstruktur (Art der Lehrgänge, Gebäudekosten) und Einzugsgebiet der einzelnen Schulen unterschiedlich hoch aus bzw. es kann sich sogar ein Nettogewinn der Standortgemeinde ergeben (z. B. Kunstgewerbeschule). Im Total sind die externen Effekte auf dem Sektor Schulen zu Ungunsten der Stadt Bern jedoch beträchtlich. 
Bei den städtischen Schulen (Untergymnasium, Gymnasium, Höhere Mädchenschule, Wirtschaftsmittelschule, Gewerbe- und Kunstgewerbeschule, Lehrwerkstätten, Frauenschule u.a.) entstand 1977 bei einem Nettoaufwand von rund 21 Mio Franken eine Nettobelastung der Stadt Bern zugunsten der Außengemeinden von 2,758 Mio Franken und zugunsten der Gebiete außerhalb der Region Bern von 3,184 Mio Franken. Bei den durch die Stadt Bern subventionierten Schulen (Kaufmännische Berufsschule, Berufsschulen für Verwaltung, für Buchhändler, für Verkaufspersonal und Ingenieurschule HTL) ergab sich bei einem Nettoaufwand von rund 4,5 Mio Franken eine Nettobelastung der Stadt Bern zugunsten der Außengemeinden von 0,515 Mio Franken und zugunsten des Gebietes außerhalb der Region Bern von 0,559 Mio Franken. Die gesamten externen Effekte auf dem Sektor Schulen beliefen sich somit 1977 auf 7,016 Mio Franken zu Lasten der Stadt Bern.

\subsection{Zusammenfassung}

Auf eine Darstellung der weiteren Infrastrukturbereiche mit externen Effekten muß hier aus Platzgründen verzichtet werden. Alle externen Kosten und Nutzen aus Infrastrukturleistungen, wie sie in der Studie berechnet wurden, sind in Tab. 3 zusammengestellt. Bei einem auf die Benutzung durch die Außengemeinden entfallenden Betrag von über 30 Mio Franken leisteten letztere nur Kostenbeiträge bzw. Gegenleistungen im Umfange von 10,1 Mio Franken. Somit ergaben sich 1977 im Total externe Kosten der Stadt Bern zu Gunsten der Außengemeinden von über 20 Mio Franken.
Im Verhältnis zum Gebiet außerhalb der Region Bern konnten Infrastrukturkosten der Stadt Bern von rund 20 Mio Franken berechnet werden bei Beiträgen von knapp 5,5 Mio Franken. Allerdings ließen sich hier die Leistungen der Stadt Bern und die Gegenleistungen des betreffenden Gebietes zu Gunsten der Stadt Bern nicht in allen Bereichen sauber berechnen (z. B. Privatverkehr), so daß die resultierenden externen Kosten/Nutzen als unvollständig zu betrachten sind. Gesamthaft resultieren aber für die Kernstadt externe Kosten aus Infrastrukturleistungen von über 30 Mio Franken jährlich.

Es erscheint als logisch, daß solche externe Effekte in irgendeiner Form korrigiert werden müssen, um langfristig das Funktionieren der Kernstädte gewährleisten zu können. Die Lösung des Problems muß aber auf der politischen Ebene gesucht werden. Es sind verschiedene Möglichkeiten denkbar wie z. B. neue kantonale Gesetze (Regelung von Beiträgen, Finanzausgleich über einen Steuerkraftausgleich hinaus), freiwillige Abmachungen zwischen der Kernstadt und den Außengemeinden oder Unterstellung einzelner Infrastruktureinrichtungen unter eine regionale Trägerschaft (Internalisierung der Spillovers) wie der Spitalverband Bern.

Tabelle 3 Stadt Bern: Zusammenstellung der externen Effekte durch Bereitstellung von Infrastrukturleistungen

\begin{tabular}{|c|c|c|c|c|c|c|c|c|c|}
\hline Infrastrukturgebiet & $\begin{array}{l}\text { Privat- } \\
\text { verkehr }\end{array}$ & $\begin{array}{l}\text { Öffentl. } \\
\text { Verkehr }\end{array}$ & Kultur & Schulen & $\begin{array}{l}\text { Naherho- } \\
\text { lung, } \\
\text { Sport- } \\
\text { anlagen }\end{array}$ & $\begin{array}{l}\text { Gesund- } \\
\text { heit }\end{array}$ & $\begin{array}{l}\text { Sicherheit, } \\
\text { Gefahren- } \\
\text { abwehr }\end{array}$ & $\begin{array}{l}\text { Versor- } \\
\text { gung, Ent- } \\
\text { sorgung }\end{array}$ & Tourismus \\
\hline Verteilungsgröße & 26,099 & 2,348 & 21,952 & 25,569 & 11,236 & • & 3,907 & 2,328 & 0,870 \\
\hline Anteil Außengemeinden & 9,918 & 1,584 & 5,816 & 8,311 & 2,116 & . & 1,091 & 1,406 & 0,121 \\
\hline Anteil außerhalb Region & 5,220 & - & 4,829 & 9,148 & $(0,594)$ & - & - & $+0,018$ & 0,117 \\
\hline \multicolumn{10}{|l|}{ Leistungen/Beiträge } \\
\hline Anteil Außengemeinden & 3,465 & 1,046 & 0,596 & 5,039 & - & . & - & - & 0,000 \\
\hline Anteil außerhalb Region & . & - & 0,040 & 5,405 & . & - & - & - & - \\
\hline \multicolumn{10}{|l|}{ Externe Effekte } \\
\hline Anteil Außengemeinden & 6,453 & 0,538 & 5,220 & 3,273 & 2,116 & $0,185^{1)}$ & 1,091 & 1,406 & 0,121 \\
\hline Anteil außerhalb Region & & - & 4,789 & 3,743 & $(0,594)$ & - & - & $+0,018$ & 0,117 \\
\hline Total & $(6,453)$ & 0,538 & 10,009 & 7,016 & $(2,710)$ & 0,185 & 1,091 & 1,388 & 0,238 \\
\hline
\end{tabular}

Alle Angaben in Mio Fr., Bezugsjahr der Zahlen: i.a. 1977 (Details siehe Studie), Zahlen in Klammern = unvollständige Werte.

1) Unter den geltenden Vereinbarungen (Spitalverband Bern) nach Auffassung der Studie ungedeckter Restbetrag.

2) Anteil außerhalb Region Bern am Reingewinn der Wasserversorgung. 


\section{Externe Effekte durch Beeinflussung des Steueraufkommens und Versuch einer Leistungsbilanz}

\subsection{Allgemeines}

Die externen Effekte zwischen Kernstadt und Außengemeinden beschränken sich nicht auf den Sektor der Infrastruktur, sondern es sind noch weitere in beiden Richtungen laufende Ströme in die Betrachtung einzubeziehen.

Die Außengemeinden profitieren vom attraktiven Stellen-, Güter- und Dienstleistungsangebot privater und öffentlicher Art sowie von den gesellschaftlichen Kontaktmöglichkeiten der Stadt; diese wiederum profitiert von der Ausweitung des Arbeitsund Absatzmarktes für die städtische Wirtschaft. Es ergeben sich direkte Wirkungen in der Form von Einkommensströmen, die das Steueraufkommen beeinflussen sowie indirekte Wirkungen durch die Verbesserung der Standortgunst.

Diese Teile der Studie sind z.Z. noch in Bearbeitung, so daß noch nicht in allen Bereichen zahlenmäßige Angaben gemacht werden können.

\subsection{Einkommensströme (Arbeitseinkommen der Pendler)}

Ziel dieses Abschnittes der Studie ist es, erstens die Struktur der außerhalb der Stadt Bern wohnenden, aber in der Stadt beschäftigten Personen (Zupendler) zu untersuchen und zweitens das Arbeitseinkommen dieser Personen für 1977 abzuschätzen. Daraus kann das entsprechende steuerbare Einkommen und der am auswärtigen Wohnsitz anfallende Steuerbetrag berechnet werden.

Detaillierte Angaben über die Struktur der in der Stadt Bern beschäftigten Personen (Wohnort, Grad der Erwerbstätigkeit, Nationalität, Stellung im Beruf, Arbeitgeber usw.) lassen sich nur den Daten der Volkszählung 1970 entnehmen. Eigens für diese Studie gemachte Auswertungen ergaben folgende Werte: 1970 wohnten rund $65 \%$ aller in der Stadt Bern Beschäftigten in der Stadt selbst, während rund $23 \%$ in den Außengemeinden und $11 \%$ außerhalb der Region Bern ihren Wohnsitz hatten. Je nach Beschäftigungsgrad, Arbeitgeber und Stellung im Beruf wichen die Anteile aber stark von diesem Gesamtwert ab. So wohnten die nur in Teilzeit beschäftigten Personen zu 76\% und die kontrollpflichtigen Ausländer zu $78 \%$ in der Stadt. Nach Arbeitgeber wurden folgende Anteile für den Wohnsitz in der Stadt Bern ermittelt: Privatwirtschaft $65 \%$, Bund $59 \%$, Kanton $66 \%$, Stadt Bern $82 \%$. Die vollbeschäftigten Berufstätigen mit Arbeitsort Bern (ohne kontrollpflichtige Ausländer) zeigten differenziert nach der Stellung im Beruf folgende Anteile für den Wohnsitz Bern: Selbständigerwerbende 79\%1), Di- rektoren und leitende Angestellte 54\%, untere Angestellte und gelernte Arbeiter $61 \%$, an- und ungelernte Arbeiter $70 \%$. Es zeigt sich somit, daß Personen mit niedrigeren Einkommen (Ausländer, Arbeiter, Teilzeitbeschäftigte) eher in der Stadt wohnen, während die höheren Einkommensklassen häufiger auswärts wohnen.

Für die Berechnung der Einkommensströme 1977 wurde von folgenden Annahmen ausgegangen: In Bern wohnhafte Berufstätige 73000 , Wegpendler von Bern 5000, Zupendler nach Bern 47000 (31 500 von Außengemeinden, 15500 von außerhalb der Region), Beschäftigte in der Stadt Bern 115000. Das Arbeitseinkommen dieser Beschäftigten wurde nun differenziert nach Erwerbsgrad, Nationalität, Arbeitgeber, Stellung im Beruf und z. T. sogar einzelnen Berufsgruppen geschätzt und nach ihrem Wohnort aufaddiert.

Die Berechnungen ergaben für 1977 ein Arbeitseinkommen von rund 1,2 Mia Franken für die Zupendler aus den Außengemeinden und von 525 Mio Franken für die Zupendler von außerhalb der Region Bern. Unter der Annahme von durchschnittlichen Abzügen und bei einem Steuersatz von 2,3 der einfachen Staatssteuer (= Gemeindesteuer der Stadt Bern 1977) resultierten Steuerbeträge aus diesen Einkommensströmen von rund 76 Mio Franken für die Pendler aus den Außengemeinden und von 30 Mio Franken für die außerhalb der Region wohnenden Beschäftigten. Von diesen Zahlen müssen noch als Gegenrechnung die Arbeitseinkommen bzw. die Steuerbeträge der Wegpendler der Stadt Bern in Abzug gebracht werden. Diese wurden unter den gleichen Annahmen wie oben auf 132 Mio Franken (Arbeitseinkommen) bzw. 8 Mio Franken (Steuerbetrag) für die Außengemeinden und 47 Mio Franken bzw. 3 Mio Franken für die Gebiete außerhalb der Region Bern geschätzt.

Aus der Beeinflussung des Steueraufkommens durch zwischengemeindliche Einkommensströme ergeben sich somit für die Stadt Bern externe Effekte von rund 68 Mio Franken zugunsten der Außengemeinden und von 27 Mio Franken zugunsten des Gebietes außerhalb der Region Bern. Diese Zahlen dürfen jedoch nicht einem Steuerentgang der Kernstadt in dieser Höhe gleichgesetzt werden, denn die 47000 Zupendler könnten mit ihren Angehörigen infolge fehlender Wohnungen nur zum geringsten Teil in der Stadt Bern Wohnsitz nehmen. Zudem müssen die Wohnsitzgemeinden aus den Steuereinnahmen für die Pendler und ihre Angehörigen Infrastrukturleistungen erbringen (Schulen, Versorgung, Entsorgung usw.).

Im weiteren müssen die oben genannten Steuerbeträge noch um die sachlichen Steuerteilungen der na-

1) Die Selbständigerwerbenden umfassen eine sehr heterogene Gruppe vom Detaillist und Handwerker bis zum Arzt und Fabrikbesitzer. 
türlichen Personen zwischen Kernstadt und Außengemeinden bzw. Gebiet außerhalb der Region Bern korrigiert werden. Im Kanton Bern versteuern die selbständigerwerbenden natürlichen Personen zwei Drittel ihres Erwerbseinkommens am Arbeitsort und ein Drittel am Wohnort.

\subsection{Konsumströme}

Ein weiteres Hauptkapitel der Studie ist die Untersuchung der Konsumströme zwischen Kernstadt, Außengemeinden und Gebiet außerhalb der Region Bern, wobei wir uns auf den Detailhandel und das Gastgewerbe beschränken. Dieser Teil der Studie wurde von einem außenstehenden Wirtschaftsfachmann für Verkaufsberatung (Dr. oec. KURT VON$\mathrm{ESCH}$, Bern) durchgeführt.

Die empirischen Untersuchungen des Detailhandels gingen von den Verkaufsflächen und Umsätzen und der verfügbaren Kaufkraft der Bevölkerung in Kernstadt und Außengemeinden aus. Die Ergebnisse der Schätzungen für das Jahr 1978 sind in Tabelle 4 zusammengestellt. Knapp die Hälfte der in der Stadt
Bern getätigten Detailhandelsumsätze kommen demnach von außerhalb, davon drei Fünftel von den Außengemeinden und zwei Fünftel vom Gebiet außerhalb der Region Bern. Etwa die Hälfte der detailgewerblichen Kaufkraft der Außengemeinden wird in der Stadt Bern verausgabt. Dagegen sind die geschätzten Kaufkraftexporte der stadtbernischen Bevölkerung in die Außengemeinden und ins Gebiet außerhalb der Region Bern relativ bescheiden.

Um die externen Effekte der Detailhandelsumsätze auf die Kernstadt abschätzen zu können, müssen nun noch das Steueraufkommen des Detailhandels und die sich aus den Kaufkraftimporten ergebenden Anteile daran untersucht werden. Diese bilden einen externen Nutzen der Stadt Bern und damit einen Gegenstrom zu den fiktiven Steuerverlusten aus den Einkommensströmen der Pendler.

Bei den Untersuchungen im Gastgewerbe stehen vor allem die Ausgaben der Pendler für die Mittagsverpflegung in der Stadt Bern im Vordergrund, die einen beträchtlichen Teil der Umsätze im Gastgewerbe der Stadt Bern und damit am Steueraufkommen dieser Branche ausmachen.

Tabelle 4 Region Bern: Umsätze und Kaufkraftströme im Detailhandel 1978

\begin{tabular}{lc}
\hline Umsätze/Kaufkraftströme Detailhandel $^{1)}$ & Mio Fr. \\
\hline Umsatz Detailhandel Stadt Bern & 1840 \\
Umsatz Detailhandel Außengemeinden & 600 \\
\hline Kaufkraftimport der Stadt Bern von Außengemeinden & 577 \\
Kaufkraftimport der Stadt Bern vom Gebiet außerhalb der Region Bern & 279 \\
Kaufkraftexport der Stadt Bern in die Außengemeinden ${ }^{2)}$ & 37 \\
Kaufkraftexport der Stadt Bern ins Gebiet außerhalb der Region Bern & 42 \\
\hline
\end{tabular}

1) Detailhandel ohne Gastronomie, Banken, Reisebüros, Auto- und Treibstoffverkäufe.

2) darunter Einkaufszentrum Shoppyland Schönbühl.

\subsection{Versuch einer Leistungsbilanz}

Die noch zu erstellende Leistungsbilanz im Verhältnis Kernstadt/Außengemeinden wird alle für die öffentliche Hand relevanten zwischengemeindlichen Ströme (externe Kosten/Nutzen) sowie die Anteile an den Gemeindesteuern aus zwischengemeindlichen Einkommens- und Konsumströmen umfassen. Diese sowie einige innerkommunale Ströme der volkswirtschaftlichen Gesamtrechnung sind in Figur 2 schematisch dargestellt. Dabei ist die Breite der Ströme bei den Arbeitseinkommen und den Konsumausgaben in etwa proportional zu den effektiven Werten. Die Infrastrukturleistungen und die Steuern mußten dagegen im Vergleich zu den Konsum- und Einkommensströmen um ein mehrfaches zu breit eingetragen werden. Unter sich sind sie aber wieder in etwa proportional zu den effektiven Beträgen. Durch schwarzen Vollton bzw. Schrägschraffur sind die positiven und negativen Effekte für die Kernstadt hervorgehoben, soweit sie überhaupt noch dargestellt werden können. Nicht mehr eingetragen werden können z. B. die sich aus den Konsumausgaben der Kernstadt in den Außengemeinden ergebenden Anteile an den Steuern der Unternehmungen in den Außengemeinden.

Die größten negativen Effekte für die Kernstadt bilden die Steueranteile aus den Arbeitseinkommen der Zupendler nach Bern und die Infrastrukturleistungen der Kernstadt für die Außengemeinden. Die größten positiven Effekte für die Kernstadt ergeben sich aus den Steueranteilen, die aus den Konsumausgaben der privaten Haushalte der Außengemeinden in der Kernstadt und aus den Arbeitseinkommen der in Bern wohnhaften Wegpendler resultieren. Gesamthaft gesehen kann aber schon im jetzigen Zeit- 


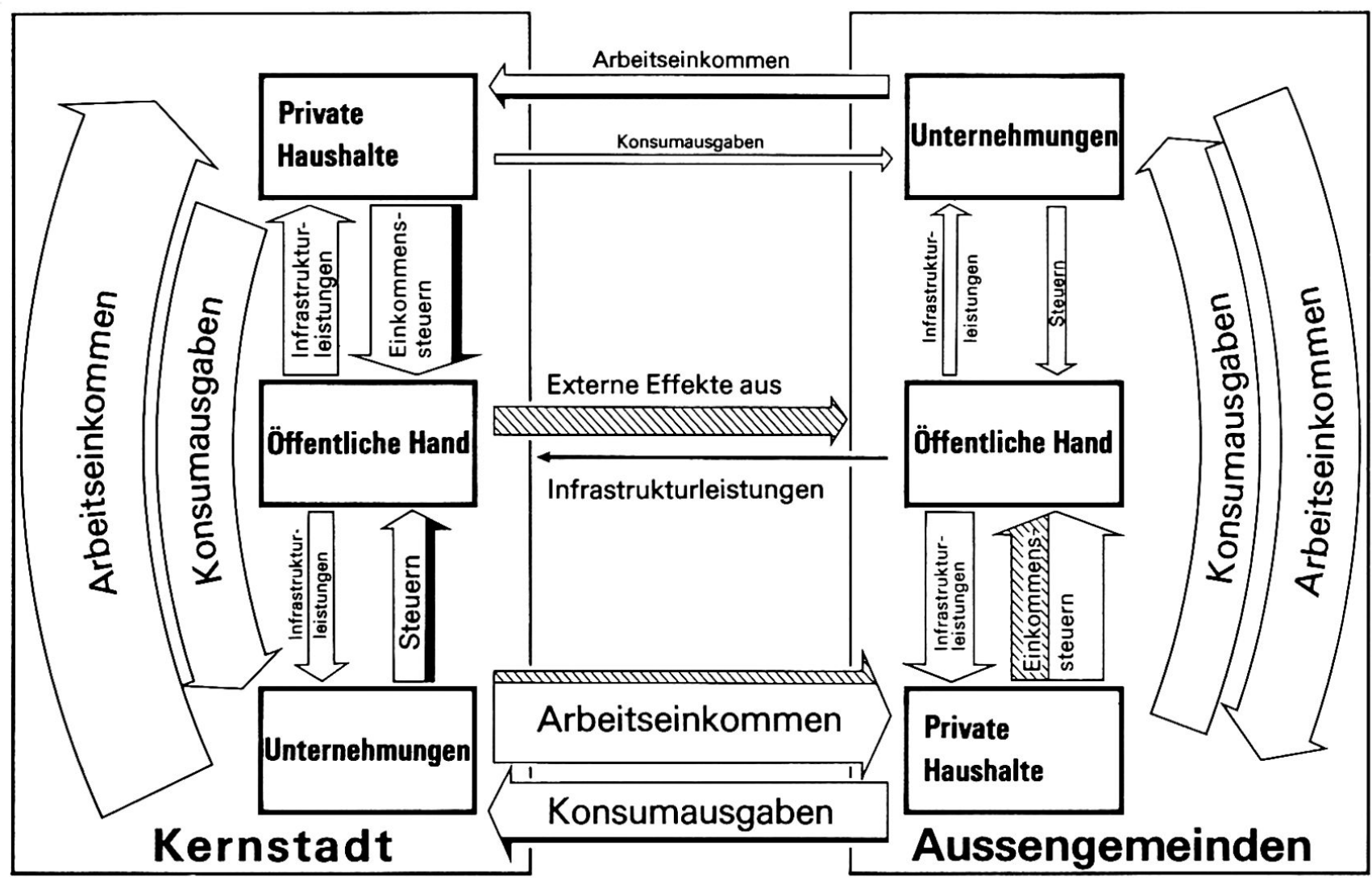

positive externe Effekte für die Kernstadt

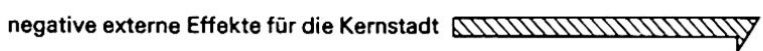

punkt ohne genaue Kenntnis aller Zahlen gesagt werden, daß die negativen externen Effekte für die Kernstadt im Falle von Bern bei weitem überwiegen.

\section{Literatur}

BUCHER, J.: Innerregionaler Finanzausgleich, ein Modell und dessen Anwendung auf die Region Bern; Berner Beiträge zur Stadt- und Regionalforschung, Heft 2/1973.

BEER, R.: Die sachlichen Steuerteilungen zwischen Gemeinden in der Region Bern, Berichte der Stadtund Regionalforschungsstelle (Refo) Bern Nr.42, 1974.
THOMET, R.: Die wirtschaftliche Belastung der Stadt Bern als Bundessitz; Refo-Berichte Nr. 49, 1975.

GABRIEL, L.: Schätzung der jährlichen Konsumausgaben der Einwohner der Gemeinde Köniz, die in der Stadt Bern getätigt werden; Schriftl. Arbeit Uni Bern 1977.

KOLLER, M.: Die Leistungen der Kernstadt und ihre Finanzierung (Zürich); Die Stadt, Heft 1/1980.

ABT, R.: Interregionale Spillovers (Vorstudie); NFP «Regionalprobleme in der Schweiz», 1980 (vervielfältigt).

STATISTISCHES AMT DER STADT BERN: Studie über die Verflechtungen der Kernstadt mit den Außengemeinden in der Region Bern, Bern 1980 (in Bearbeitung). 